\title{
Mycotic Infection (Fungal Ball) of the Paranasal Sinuses: A Clinicopathological Observation
}

\author{
Vladimír Bartoš ${ }^{1^{*}}(\mathbb{D}$, Veronika Bartošová²
}

\begin{abstract}
Mycotic infections of the nasal cavity and paranasal sinuses represent a wide spectrum of disorders that vary in clinical presentation, histopathologic appearances, and biological significance. The second most common form is a fungal ball.

The objective of the research was to describe the cases of paranasal sinus fungal ball found in the files of patients with chronic rhinosinusitis.

Materials and Methods. There were demonstrated clinical and histopathological data of two patients.

Results. A 42-year-old woman and a 40-year-old man with chronic hypertrophic sinusitis had a large amount of friable cheesy mass in the maxillary sinus. The female patient previously underwent upper dental arch sanation. Histology revealed dense laminated masses of matted fungal hyphae that were separate from the mucosa. Sinonasal mucosa showed mild chronic inflammation (male) and severe non-specific chronic active inflammation (female). No evidence of fungal invasion in the mucosa was found.

Conclusions. Fungal ball represents a non-invasive form of mycotic rhinosinusitis with favourable prognosis. Biopsy examination plays an important role in the diagnostic process. Although the diagnosis can also be achieved by polymerase chain reaction assays, biopsy is the only way to explore the status of an inflammatory damage to adjacent tissue and, thus, to rule out potential invasive mycotic sinus disease with much worse clinical outcome.
\end{abstract}

\section{Keywords}

Mycotic Infections; Fungal Ball; Chronic Rhinosinusitis

${ }^{1}$ Department of Pathology, Martin's Biopsy Center, Ltd., Martin, Slovakia

${ }^{2}$ Clinic of Infectology and Travel Medicine, Jessenius Faculty of Medicine, Martin, Slovakia

*Corresponding author: vladim.bartos@gmail.com

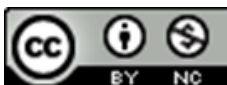

Copyright @Vladimír Bartoš, Veronika Bartošová, 2020

\section{Background}

Fungal (mycotic) infections represent one of the four major microbiological subgroups (bacteria, viruses, parasites, fungi) of human diseases [1]. Although there are thousands of different types of fungi, there are only a small number of fungi being clinically relevant. Among them, the most common species in medical practice are Candida and Aspergillus species [1]. Fungal microorganisms are abundant in the air and those that are inhaled form a part of the normal sinonasal flora. In normal situation, they are destroyed by immunological mechanisms and do not cause any symptoms [1]. Under the circumstances when the immunological pathways are disrupted, they result in clinically apparent pathological conditions [1]. Mycotic infections of the nasal cavity and paranasal sinuses (fungal rhi- nosinusitis (FRS) are a spectrum of disorders rather than one distinct entity. They vary in clinical presentation, histopathologic appearances, and biological significance [1-5]. FRS is traditionally classified as non-invasive or invasive based on whether fungi have invaded into tissue or not $[1,3,4,6]$. Both groups are further divided into three subtypes (Table 1).

Their detailed description would go far beyond the scope of this article, but they are well described in another papers [14]. Briefly, saprofytic fungal infestation means an asymptomatic fungal colonization of the secretions of the sinonasal cavity that results in inflamed and ulcerated/crusted mucosa. Fungal balls are an extramucosal, densely matted conglomeration of fungal hyphae usually associated with minimal mucosal inflammation [1, 3, 4]. Allergic FRS is the most common form of fungal sinonasal disease occuring particu- 
Table 1. Traditional classification of mycotic rhinosinusitis (loosely adapted from ref. [2]).

\begin{tabular}{lccc}
\hline Category & Host immune status & Role of fungus & Clinical course \\
\hline & A. Non-invasive (no invasion into the mucosa) \\
\hline 1. Saprobic infestation & immunocompetent & saprobe & frequently asymptomatic \\
2. Fungal ball & immunocompetent & saprobe & chronic \\
3. Allergic & atopic & allergen & chronic \\
\hline \multicolumn{4}{c}{ B. Invasive (invasion into the mucosa) } \\
\hline 1. Acute & immunocompromised & pathogen & acute, rapid onset \\
2. Chronic & immunocompromised & pathogen & chronic \\
3. Granulomatous & immunocompetent & pathogen & indolent, chronic \\
\hline
\end{tabular}

larly in immunocompetent, atopic patients. It is rather a result of an inflammatory reaction toward fungi than a true mycotic infection [1,3]. Microscopy demonstrates mucin with a large amount of eosinophils, eosinophilic debris, CharcotLeyden crystals, and other inflammatory cells arranged in a laminar pattern and associated with rare, scattered fungal hyphae $[1,3,4]$. Acute invasive FRS is a destructive form of sinonasal fungal disease occuring in patients with immunocompromised status, which is characterized by a rapid onset and an aggressive clinical course. Histology shows angioinvasion of fungal forms resulting in vascular thrombosis. Chronic invasive FRS is a slowly destructive process characterized by dense accumulation of fungal microorganisms in the sinonasal mucosa with occasional vascular invasion. This entity occurs in the background of acquired immunodeficiency [1, 3, 4]. Chronic granulomatous invasive FRS is seen in immunocompetent patients. It is characterized by the presence of noncaseating granulomas with Langhans-type giant cells with extensive fibrosis, and only scanty fungal hyphae [1, 3, 4]. In addition to that classification scheme, Korean authors [7] have recently proposed a new subtype of FRS, i.e., microinvasive fungal rhinosinusitis, which could be included in the classification as a separate entity in the future.

The objective of this paper was to demonstrate the cases of paranasal sinus fungal ball found in the files of patients with chronic rhinosinusitis and to discuss the clinicopathological aspects of disease.

\section{Materials and Methods}

A retrospective analysis of all consecutive cases of chronic rhinosinusitis that were histologically diagnosed at the Martin's Biopsy Center in Martin (Slovakia) during a one-year period (July 2019 - June 2020) was carried out. All participants were registered in the Pathology Archive Computer Program, from which the required histopathological data were extracted. The cases manifesting with a fungal ball have been searched for from this file. Clinical data of the patients needed for the study were obtained from their medical records. Biopsy samples were processed in the formalin-fixed paraffin-embedded tissue blocks which were then stained with hematoxylin and eosin (H\&E). Selected cases were also investigated with special staining methods for detection of fungal microorganisms, such as Periodic Acid-Schiff (PAS) stain, Grocott's methenamine silver stain, and Gram stain.

\section{Results}

In the study period, a total of 88 individuals with diagnosis of chronic rhinosinusitis or inflammatory polyp of the sinonasal mucosa were found in the database. Among them, two (2.2\%) individuals presented with fungal ball. Next, we provide the clinicopathological findings of these patients.

\section{Clinical Data}

The first patient was a 42-year-old woman, who suffered from pressure-induced dull pain in both sides of maxillary sinus area and difficulty breathing through the nose in the cold.

She claimed that the problems had been present for a long time. In the past, she underwent upper dental arch sanation. $\mathrm{X}$-ray imaging and computed tomography (CT) showed mucosal hypertrophy and a polypoid degeneration of the musoca in both maxillary sinuses (more pronounced in the right one) with thickening of the walls and obstruction of the ostiomeatal complex. In addition, heterogeneous opacities were visible in the antrum of Highmore. Nasal cultures grew Streptococcus pneumoniae. A diagnosis of chronic hypertrophic rhinosinusitis with purulent secretion was made. Patient had taken several courses of local antibiotics (Zinnat, Biseptol, Pamycon), but did not have any relief and the condition continued to deteriorate. The last time, she observed mucus discharge from the nose. Control CT scan confirmed a worse local finding as compared to the previous one. Therefore, functional endoscopic sinus surgery was performed. During the operation, both maxillary sinuses (more in the right side) were filled with mucopurulent cheesy mass, that resembled fungal balls. The mucosa was edematous and hypertrophic and both ostiomeatal units were obturated by mucosal polyps. The pathologic material was removed and, together with resected fragments of the polypoid mucosa, sent for biopsy examination.

The second patient was a 40-year-old man, who also presented with history of difficulty breathing through the nose for the past years. There was no other significant medical history. Nasal endoscopy and CT scan of the paranasal sinuses revealed the attributes of left-sided chronic maxillary 


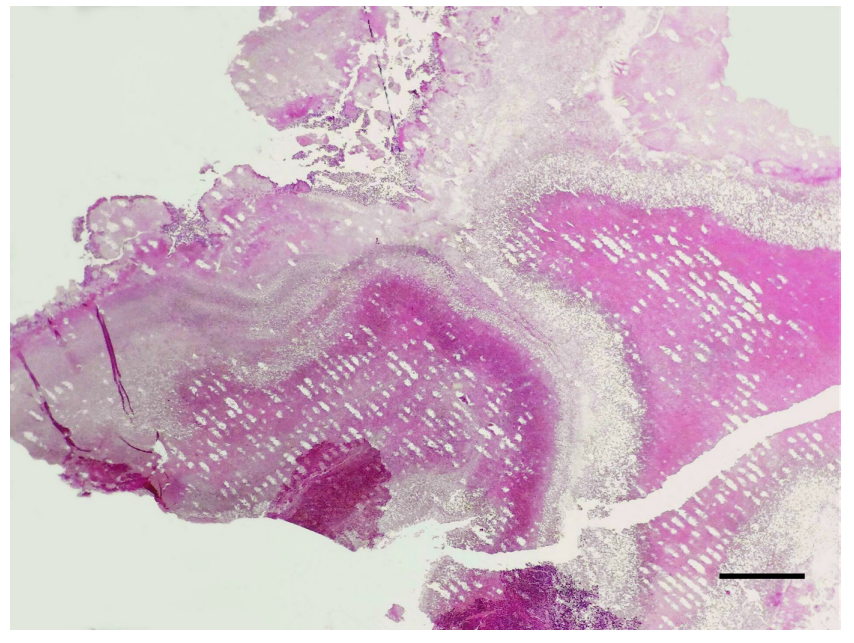

Figure 1. Fungal ball composed of a laminated mass of entangled fungal hyphae. (H\&E, original magnification 10x, scale bar $500 \mu \mathrm{m})$

rhinosinusitis with nasal septum deviation and hyperplasia of the left inferior nasal concha. The patient underwent functional endoscopic sinus surgery. Grossly, a large amount of soft friable brownish-green matter in the left sinus of Highmore was found. It looked like fungal balls. They were completely evacuated and, alongside with the parts of hyperplastic mucosa, sent for histology.

\section{Histopathological Findings}

In both patients, the histopathological findings were nearly identical. Hematoxylin and eosin stained sections revealed dense laminated masses of matted fungal hyphae embedded in fibrinous, necrotic exudate (Fig. 1).

These conglomerations were completely separate from the sinus mucosa (Fig. 2).

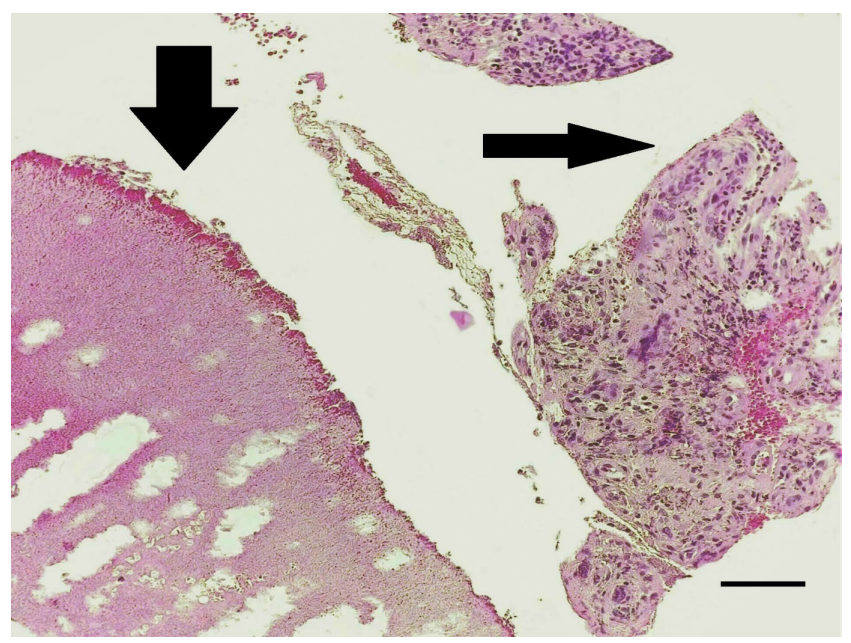

Figure 2. Fungal ball (thick arrow) completely separated from the mucosa (thin arrow). (H\&E, original magnification 20x, scale bar $250 \mu \mathrm{m}$ )
A high-power magnification identified multiple entangled septate acute-angle branching fungal hyphal elements. They were Grocott's stain positive (Fig. 3), PAS stain positive (Fig. 4) and Gram stain positive (Fig. 5).

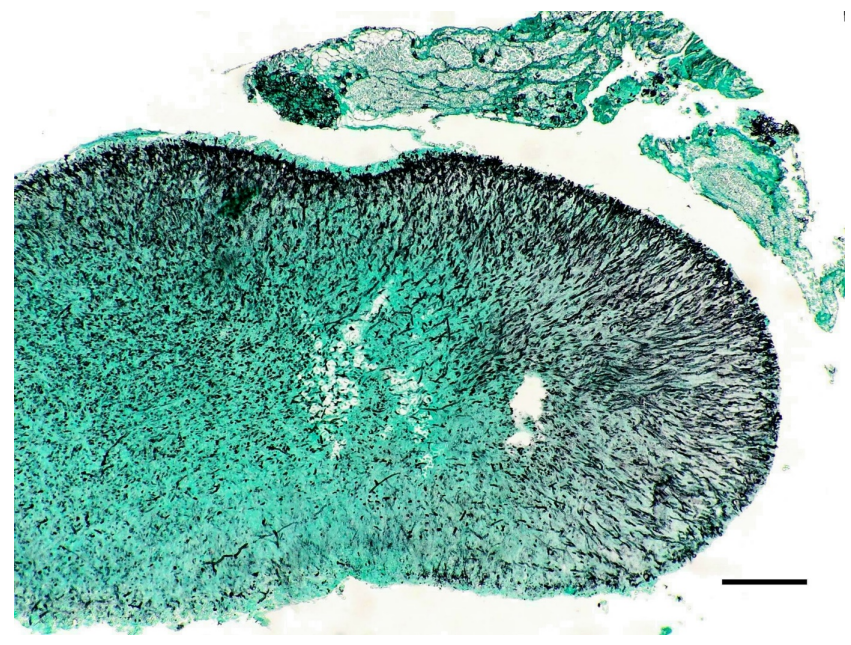

Figure 3. Grocott's methenamine silver stain highlighting fungal hyphae (black color). (Original magnification 20x, scale bar $250 \mu \mathrm{m})$

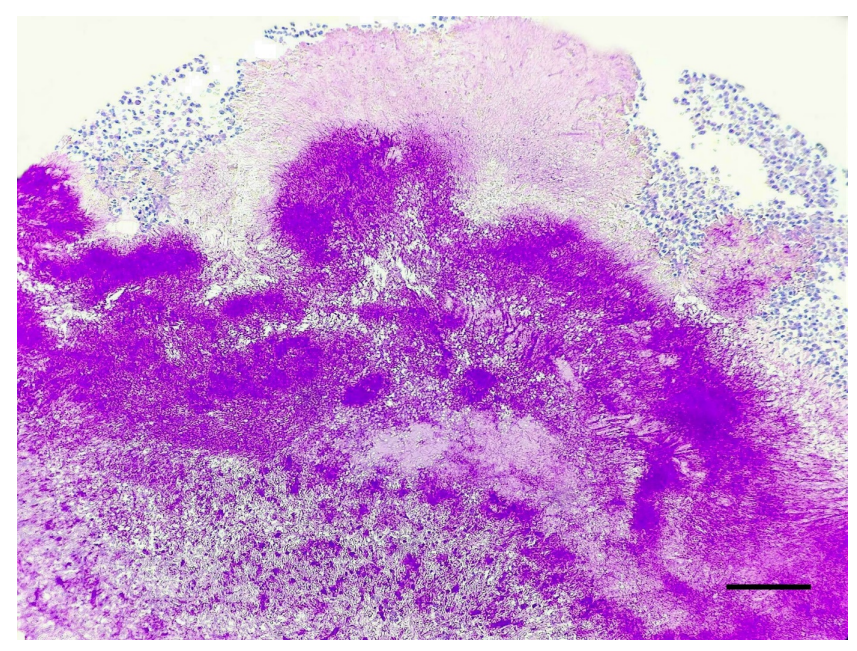

Figure 4. PAS stain highlighting fungal hyphae (red-purple color). (Original magnification 40x, scale bar $125 \mu \mathrm{m}$ )

In the surrounding mucosa, mild chronic inflammatory reaction (male patient) and severe non-specific chronic active inflammation (female patient) (Fig. 6) were visible.

No predominance of eosinophils, allergic mucin, or granulomatous reaction was found. There was no evidence of fungal invasion in the mucosa. The findings were consistent with a diagnosis of maxillary sinus fungal ball.

Both patients have not undergone a control medical checkup within 9 months after surgery, when this article was written. 


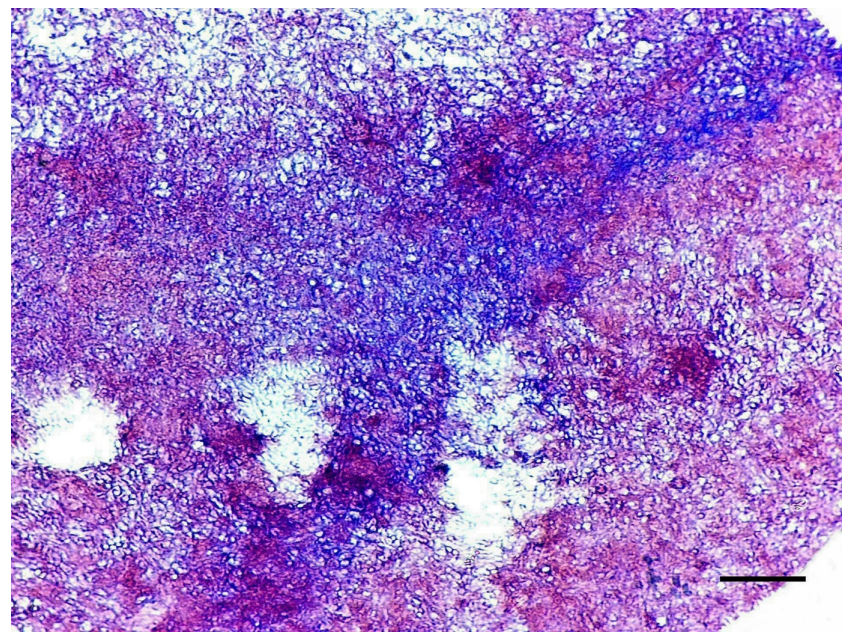

Figure 5. Gram stain highlighting fungal hyphae (blue color). (Original magnification 100x, scale bar $50 \mu \mathrm{m}$ )

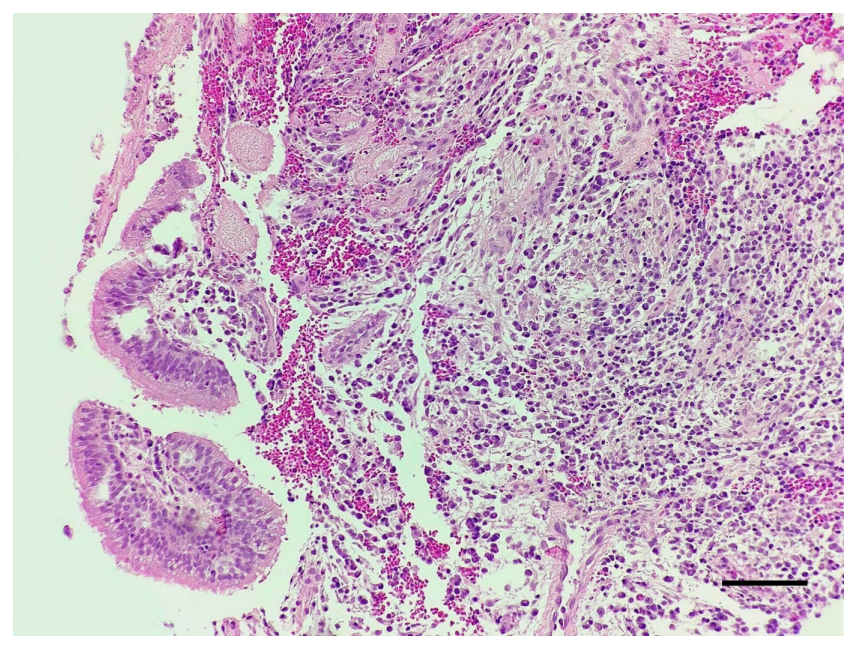

Figure 6. Massive inflammation of sinus mucosa without an evidence of mycotic organisms. (Original magnification 40x, scale bar $125 \mu \mathrm{m})$

\section{Discussion}

Fungal ball is the second most common form of FRS, comprising about $40 \%$ of all cases [8]. It represents a rare and specific form of mycotic infection of the sinonasal cavity. The terms "mycetoma" and "aspergilloma" were previously used for this entity [1-4]. Although, in the literature, to designate the sinonasal fungal ball, they were used interchangeably, in fact, both of them were misleading. "Mycetoma" is a chronic local invasion of subcutaneous tissue by bacteria or fungi with the formation of a sinus tract, swelling, and granules [24]. The general term "aspergilloma" is incorrect as well, as the disease is not always due to Aspergillus species [1-4]. Therefore, a fungal ball was considered to be the most appropriate term [1-4]. In accordance with our observations, fungal balls frequently occur in only one sinus and the maxillary sinus is most commonly affected $[1-6,8-10]$. The reasons for this phenomenon are unknown [5]. Typically, they occur in immunocompetent patients and are reported to be more prevalent among middle-aged and elderly females $[1,3,4,6,9,10]$. The pathogenesis of fungal ball formation is not completely understood [6]. It is widely accepted that the disease begins with the inhalation of fungal spores that then become sequestered in a specific location [1]. Prior surgery or mucosal injury is a risk factor $[1,4]$. In particular, there is a strong association of maxillary fungal balls with previous dental treatment. Even our female patient underwent dental intervention. Clinical presentation of fungal ball is usually non-specific and may be asymptomatic [1]. This is often encountered as a part of investigation and treatment for conventional chronic rhinosinusitis. The main clinical suspicion toward the diagnosis are cheesy and clay-like inspissated mucous masses found on nasal endoscopy or intraoperativelly [1]. Fungal balls are easily identifiable in the light microscope, as they have typical histomorphology described above. The use of special staining techniques, such as Grocott's or PAS methods reliably supports the final diagnosis. However, it is important to note that microbiological cultures are unreliable when it comes to fungal balls [1]. In approximately $70 \%$ of the cases, the diagnosis is made exclusively by histology, while the cultures are negative [3]. In some studies [8, 11, 12], the positive rates of fungal culture in paranasal fungal ball ranged between $22.6 \%$ $-51 \%$. This may be linked to the poor viability of the fungal hyphae. In culture-positive cases, the most commonly isolated pathogen is Aspergillus species [1, 4, 5, 8, 11]. However, as the fungal colonisation leads to bacterial microbiota dysbiosis, the coexistence of many types of bacteria can be identified in mycotic rhinosinusitis [5]. Treatment of the disease consists in surgical removal of the mycotic material without the need for topical or systemic antifungal therapy [1, 4].

\section{Conclusions}

Fungal ball represents a non-invasive form of mycotic rhinosinusitis with favourable prognosis. Biopsy examination plays an important role in the diagnostic process. Although a diagnosis can also be achieved by real-time PCR assays, biopsy is the only way to explore the status of an inflammatory damage to adjacent tissue. Therefore, as a part of surgery, it is important to take a sample from surrounding mucosa to rule out potential invasive mycotic sinus disease with much worse clinical outcome.

\section{Ethical Statement}

The study was conducted according to WHO Declaration of Helsinki - Ethical Principles for Medical Research Involving Human Subjects.

\section{Informed Consent}

Informed consent was obtained from the patients. 


\section{Conflict of Interest}

The authors declare that no conflicts exist.

\section{Financial Disclosure}

The authors declared no financial support.

\section{References}

[1] Deutsch, Whittaker, Prasad. Invasive and Non-Invasive Fungal Rhinosinusitis-A Review and Update of the Evidence. Medicina [Internet]. 2019 Jun 28;55(7):319. Available from: https://doi.org/10.3390/medicina55070319

[2] Chakrabarti A, Das A, Panda NK. Controversies surrounding the categorization of fungal sinusitis. Medical Mycology [Internet]. 2009 Jan;47(s1):S299-S308. Available from: https://doi.org/10.1080/13693780802213357

[3] Chakrabarti A, Denning DW, Ferguson BJ, Ponikau J, Buzina W, Kita H, et al. Fungal rhinosinusitis. The Laryngoscope [Internet]. 2009 Jun 18;119(9):1809-1818. Available from: https://doi.org/10.1002/lary.20520

[4] Montone KT. Pathology of Fungal Rhinosinusitis: A Review. Head and Neck Pathology [Internet]. 2016 Feb 1;10(1):40-46. Available from: https://doi.org/10.1007/s12105-016-0690-0

[5] Lu Y-T, Wang S-H, Liou M-L, Shen T-A, Lu Y$\mathrm{C}$, Hsin $\mathrm{C}-\mathrm{H}$, et al. Microbiota Dysbiosis in Fungal Rhinosinusitis. Journal of Clinical Medicine [Internet]. 2019 Nov 14;8(11):1973. Available from: https://doi.org/10.3390/jcm8111973

[6] Wang L-L, Chen F-J, Yang L-S, Li J-E. Analysis of pathogenetic process of fungal rhinosinusitis: Report of two cases. World Journal of Clinical Cases [Internet]. 2020 Jan 26;8(2):451-463. Available from: https://doi.org/10.12998/wjcc.v8.i2.451

[7] Seo MY, Seok H, Lee SH, Choi JE, Hong SD, Chung S-K, et al. Microinvasive Fungal Rhinosinusitis: Proposal of a New Subtype in the Classification. Journal of Clinical
Medicine [Internet]. 2020 Feb 24;9(2):600. Available from: https://doi.org/10.3390/jcm9020600

[8] Montone KT, Livolsi VA, Feldman MD, Palmer J, Chiu AG, Lanza DC, et al. Fungal Rhinosinusitis: A Retrospective Microbiologic and Pathologic Review of 400 Patients at a Single University Medical Center. International Journal of Otolaryngology [Internet]. 2012;2012:1-9. Available from: https://doi.org/10.1155/2012/684835

[9] Kim DW, Kim YM, Min J-Y, Kim J-W, Kim J-K, Mo $\mathrm{J}-\mathrm{H}$, et al. Clinicopathologic characteristics of paranasal sinus fungus ball: retrospective, multicenter study in Korea. European Archives of Oto-Rhino-Laryngology [Internet]. 2019 Nov 28;277(3):761-765. Available from: https://doi.org/10.1007/s00405-019-05738-5

[10] Shcherbakov D, Klimova N, Malysheva T, Shcherbakova A. Large Fungal Ball of the Paranasal Sinuses and Nasal Cavity: Two Case Reports. International Journal of Biomedicine [Internet]. 2019 Dec 15;9(4):376-378. Available from: https://doi.org/10.21103/Article9(4)_CR1

${ }^{[11]}$ Liu X, Liu C, Wei H, He S, Dong S, Zhou B, et al. A retrospective analysis of 1,717 paranasal sinus fungus ball cases from 2008 to 2017. The Laryngoscope [Internet]. 2019 Aug 29;130(1):75-79. Available from: https://doi.org/10.1002/lary.27869

[12] Dufour X, Kauffmann-Lacroix C, Ferrie JC, Goujon JM, Rodier MH, Klossek JM. Paranasal sinus fungus ball: epidemiology, clinical features and diagnosis. A retrospective analysis of 173 cases from a single medical center in France, 1989-2002. Medical Mycology [Internet]. 2006 Jan;44(1):61-67. Available from: https://doi.org/10.1080/13693780500235728

Received: $2020-09-18$

Revised: 2020-10-21

Accepted: 2020-10-29 